\title{
What is the role of radiotherapy for extensive-stage small cell lung cancer in the immunotherapy era?
}

\author{
Eric G. Nesbit ${ }^{1}$, Ticiana A. Leal ${ }^{2}$, Tim J. Kruser ${ }^{1}$ \\ ${ }^{1}$ Department of Radiation Oncology, Feinberg School of Medicine, Northwestern University, Chicago, IL, USA; ${ }^{2}$ Division of Hematology \& \\ Oncology, University of Wisconsin Carbone Cancer Center, Madison, WI, USA \\ Contributions: (I) Conception and design: EG Nesbit, TJ Kruser; (II) Administrative support: TJ Kruser; (III) Provision of study materials or patients: \\ All authors; (IV) Collection and assembly of data: All authors; (V) Data analysis and interpretation: All authors; (VI) Manuscript writing: All authors; \\ (VII) Final approval of manuscript: All authors. \\ Correspondence to: Tim J. Kruser, MD. Department of Radiation Oncology, Feinberg School of Medicine, Northwestern University, 251 East Huron \\ Street, Galter Pavilion LC-178, Chicago, IL 60611, USA. Email: tkruser@nm.org.
}

\begin{abstract}
Small cell lung cancer has been a difficult disease to treat with poor survival and few significant improvements in outcomes in the last three decades. Most recently the addition of atezolizumab to chemotherapy in the first-line treatment of extensive-stage small cell lung cancer (ES-SCLC) resulted in improved overall survival and progression-free survival compared to chemotherapy alone. Recent randomized studies examining both consolidative thoracic radiotherapy and prophylactic cranial irradiation (PCI) in ES-SCLC have impacted the utilization of these interventions. The approval of immune checkpoint inhibitors (ICIs) to platinum/etoposide chemotherapy for the treatment of ES-SCLC in the front-line setting may also further impact the role of radiotherapy in this disease. In this article, we review the current evidence supporting thoracic radiotherapy in ES-SCLC and discuss the promising therapeutic implications of thoracic radiation in light of the inclusion of ICIs. We also address how the increasing routine use of surveillance brain magnetic resonance imaging (MRI) and ICIs may diminish the use of PCI in ES-SCLC.
\end{abstract}

Keywords: Immunotherapy; radiotherapy; small cell lung cancer (SCLC)

Submitted Apr 10, 2019. Accepted for publication Apr 29, 2019.

doi: 10.21037/tlcr.2019.05.01

View this article at: http://dx.doi.org/10.21037/tlcr.2019.05.01

\section{Introduction}

Recently the addition of immune checkpoint inhibitors (ICIs) into the upfront treatment of extensive-stage small cell lung cancer (ES-SCLC) was shown to improve survival in unselected patients (1). Prior to this advancement, treatment of ES-SCLC included standard of care platinumdoublet chemotherapy, with consideration of consolidative thoracic radiotherapy (RT) and prophylactic cranial irradiation (PCI) in patients who achieved response (2-4). The addition of both thoracic and brain-directed radiotherapy is supported by randomized clinical trial data, but patient and tumor factors often drive the utilization of $\mathrm{RT}$ in an individual patient. In this review, we examine how the incorporation of ICIs into the treatment paradigm may impact the enthusiasm for consolidative thoracic RT and PCI in ES-SCLC.

\section{Immunotherapy in ES-SCLC}

SCLC is characterized by frequent loss of tumor suppressors $\operatorname{TP} 53(5,6)$ and RB1 and results in an aggressive, highly complex malignancy at the molecular level with a large number of mutations present in each tumor (7). A number of studies have suggested that tumor mutational burden is associated with benefit from immunotherapy (8). SCLC carries one of the strongest associations with tobacco carcinogenesis and highest mutational burdens of any malignancy, and therefore is considered ideal for consideration of immunotherapy (7). 
Ipilimumab, an anti-cytotoxic T-lymphocyte-associated protein 4 (CTLA-4) monoclonal antibody, was the first ICI investigated in ES-SCLC in combination with platinum doublet chemotherapy. In a phase II study, patients with untreated ES-SCLC were randomized to three arms: carboplatin/paclitaxel alone, ipilimumab administered concurrently with carboplatin/paclitaxel for 2 cycles followed by chemotherapy, and phased combination of ipilimumab starting with cycle 3 of chemotherapy (9). The immune related progression-free survival (irPFS) was significantly prolonged for the phased ipilimumab arm (5.7 vs. 6.4 vs. 5.3 months; HR 0.75, $\mathrm{P}=0.11$ for concurrent and HR 0.64, $\mathrm{P}=0.03$ for phased compared to control). This signal of efficacy was accompanied by higher rates of grade $3 / 4$ toxicities in the ipilimumab arms (30\% control vs. $43 \%$ concurrent vs. $50 \%$ phased). The subsequent phase III study assigned 1,132 patients with untreated ES-SCLC to receive platinum (cisplatin or carboplatin) and etoposide plus ipilimumab $10 \mathrm{mg} / \mathrm{kg}$ or placebo every 3 weeks for a total of four doses, followed by ipilimumab or placebo maintenance every 12 weeks. This study did not meet its primary endpoint with median OS of 11.0 months for chemotherapy plus ipilimumab vs. 10.9 months for chemotherapy plus placebo [hazard ratio (HR), 0.94 ; $95 \%$ CI: 0.81 to 1.09 ; $\mathrm{P}=0.3775]$. However, it did demonstrate the feasibility of combining chemotherapy and ICI in SCLC and led the way to development of strategies investigating anti-programmed death-1 (PD-1) or anti-programmed death ligand 1 (PD-L1) antibodies in this disease (10).

In CheckMate 032, a large phase I/II study, patients with pre-treated SCLC were enrolled to either a nonrandomized cohort or a randomized cohort and were treated with nivolumab (anti-PD1 antibody) alone $3 \mathrm{mg} / \mathrm{kg}$ Q2 weeks or nivolumab $1 \mathrm{mg} / \mathrm{kg}+$ ipilimumab $3 \mathrm{mg} / \mathrm{kg}$ Q3 weeks for four cycles, followed by nivolumab monotherapy $3 \mathrm{mg} / \mathrm{kg}$ Q2 weeks, until progression of unacceptable toxicity $(11,12)$. The overall response rate (ORR) was $11 \%$ and $22 \%$, for nivolumab monotherapy and nivolumab + ipilimumab, respectively. The rate of grade $3 / 4$ toxicities was $12 \%$ and $37 \%$. This led to the inclusion of nivolumab \pm ipilimumab in the NCCN guidelines for relapsed SCLC (13). Among responders, responses were durable in the nivolumab monotherapy arm ( $\geq 6$ months in $77 \%, \geq 12$ months in $62 \%$, and $\geq 18$ months in $39 \%$ ). The durability of response was considered promising with resultant accelerated approval by the Food and Drug Administration (FDA) of nivolumab for patients with ES-SCLC with progression after platinum-based chemotherapy and at least one other line of therapy (14). Pembrolizumab has also been included in the NCCN guidelines for relapsed SCLC (13) based on the pooled analysis of the phase Ib study KEYNOTE-028 and phase II study KEYNOTE-158, which reported ORR of $33 \%$ and $19 \%$ in patients with ES-SCLC, respectively $(15,16)$.

In the second-line setting, the phase III study (CheckMate 331) investigating nivolumab vs. topotecan or amrubicin did not meet the primary endpoint of OS (17). Similarly, the phase II randomized study of atezolizumab $v s$. chemotherapy (topotecan or re-challenge platinum/ etoposide) in the second-line setting also did not meet its primary endpoint of ORR at 6 weeks (18). The OS likewise did not differ between the two arms: median OS was $9.5 \mathrm{vs.}$ 8.7 months in the atezolizumab and chemotherapy arms, respectively (adjusted HR of atezolizumab 0.84 , 95\% CI: $0.45-1.58, \mathrm{P}=0.60)$. Only $2 \%$ of the evaluable specimens had positive PD-L1 staining (SP142 clone). Of note, the 1 -year survival rate was $42.5 \%$, suggesting that there is a subset of patients that seem to derive benefit; however, no predictive clinical factors or biomarkers have been yet been identified.

Disappointingly, ICIs have not shown a significant advantage in the maintenance setting. In a phase II study, pembrolizumab $200 \mathrm{mg}$ IV every 3 weeks was given as maintenance therapy after first-line platinum doublet chemotherapy in 45 patients (19). Median PFS was 1.4 months and therefore did not improve median PFS compared with the historical data. Most recently, CheckMate 451, a phase III study in the maintenance setting, also failed to show OS benefit of ICIs $v s$. placebo (NCT02538666) (20).

The strategy of combining platinum/doublet and the PD-L1 inhibitor atezolizumab has demonstrated improved outcomes in the front-line setting. IMpower 133 was a phase 1/3 double-blind placebo-controlled trial evaluating safety and efficacy of carboplatin and etoposide in combination with atezolizumab $v s$. placebo in patients with untreated ES-SCLC (1). This trial enrolled 403 patients with ECOG PS of 0 or 1 and no symptomatic CNS disease. Co-primary endpoints were OS and investigator assessed PFS in the intention to treat population. At the primary analysis, with a median follow up of 13.9 months, median OS was 12.3 months in the atezolizumab arm $v s$. 10.3 (HR 0.70, $\mathrm{P}=0.0069$ ). The 1 -year OS was $51.7 \%$ vs. $38.2 \%$, respectively. There was also a statistically significant 1-month increase in median PFS from 4.3 to 5.2 months, and more than doubling of the 12 -month 


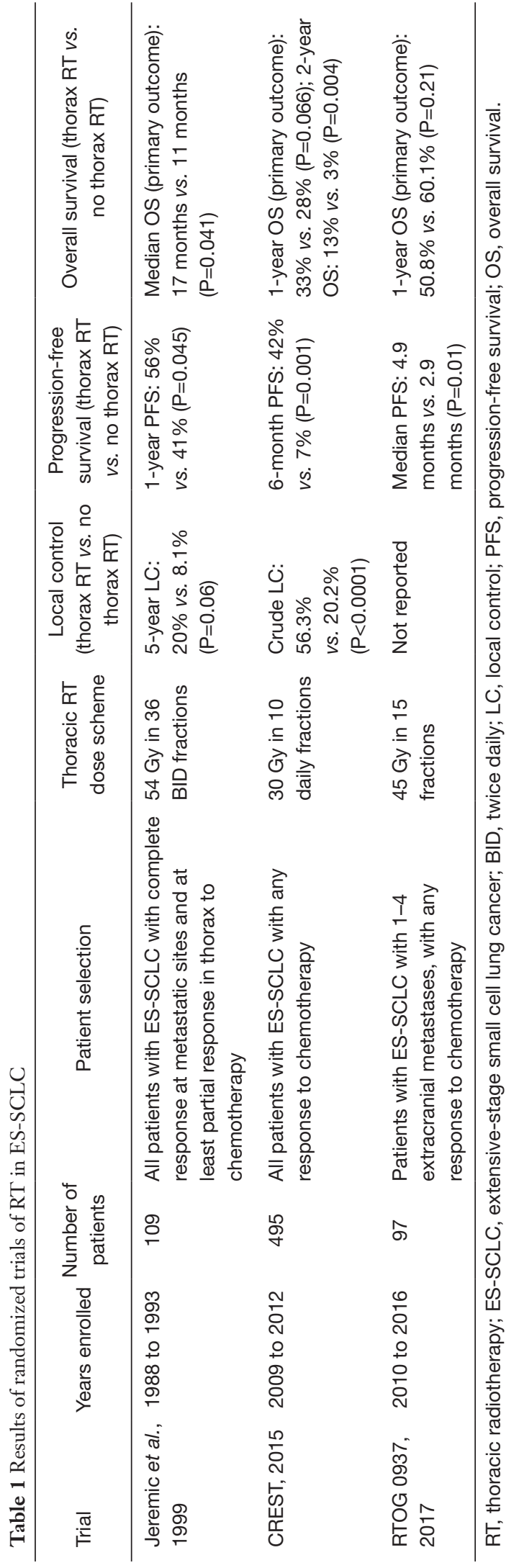

PFS rate from 5.4 to $12.6 \%$. The combination was well tolerated with no unexpected safety signals, and no reduction in chemotherapy intensity or cumulative dose in the combination therapy arm. In a subgroup analysis, blood TMB was evaluated and did not correlate with outcomes. The patient-reported outcomes showed that the addition of atezolizumab to chemotherapy was associated with health-related quality of life improvements and did not contribute to worsening toxicity or symptom burden (21). This regimen is now included in the NCCN guidelines (category 1, preferred) (13) and has received FDA approval (22).

It is clear based on the results of the IMpower 133 that immunotherapy in combination with chemotherapy has a role in the treatment of patients with untreated ESSCLC. However, many questions remain. Importantly, in this study, consolidative thoracic RT was not allowed. The number of patients who received palliative thoracic RT was very low and no unexpected adverse events were observed. PCI was permitted, however only 22 patients in each group received PCI. In the patients who received PCI, CNS-related events appeared to be more common in the atezolizumab arm. However, number of events were low and causal relationship at this time is unclear (23).

We will further examine the evidence for consolidative thoracic RT and PCI and discuss the promising strategies incorporating RT in the setting of ICI and challenges ahead.

\section{Thoracic RT in ES-SCLC}

The evidence supporting the use of consolidative thoracic RT for patients with ES-SCLC came primarily from two randomized trials (Table 1). A Yugoslavian trial enrolled 210 patients with ES-SCLC with three cycles of cisplatin/ etoposide chemotherapy (2). Patients who had a complete response at distant sites and at least a partial response at the primary site $(\mathrm{n}=109)$ were randomized to either accelerated hyperfractionated thoracic RT (54 Gy in 36 twice-daily fractions) in combination with chemotherapy followed by 2 additional cycles of chemotherapy $v s .4$ additional cycles of chemotherapy alone. Patients in both groups also received PCI, 25 Gy in 10 fractions. The thoracic RT arm appeared to offer improved local control, as well as improved survival compared to the chemotherapy alone group, with a median survival of $17 v s .11$ months $(\mathrm{P}=0.041)$. However, thoracic RT was also associated with higher rates grade $\geq 3$ esophageal toxicity, seen in $27 \%$ of the patients 
receiving accelerated hyperfractionated RT. Although some pulmonary toxicity was seen in the RT arm (5\% with grade 3 toxicity), it was not statistically significantly higher than the systemic therapy alone arm.

Despite these data indicating a survival benefit, thoracic RT for ES-SCLC was not routinely incorporated into practice due to concerns that this was a small, single institution study that utilized a non-standard chemoradiation regimen (24). Thereafter, additional data including retrospective studies $(25,26)$ and a non-randomized trial $(27)$ lent further support for the benefit of thoracic RT in ES-SCLC. The CREST trial, a Dutch phase III study, was therefore designed to more definitively answer this question, treating 495 patients with ES-SCLC with any response following standard platinum/etoposide chemotherapy to PCI and thoracic RT to 30 Gy in 10 fractions, vs. PCI alone (4). The pre-specified primary outcome of 1-year OS was not significantly different between the two arms (33\% for the thoracic RT arm, 28\% for the control group; $\mathrm{P}=0.066)$. However, the analysis of 2 -year OS found improved survival in the thoracic RT group, $13 \%$ vs. $3 \%(\mathrm{P}=0.004)$, and no severe toxicity was seen with the addition of thoracic RT. The negative pre-specified primary outcome in this trial has led to hesitancy to make thoracic RT in ES-SCLC a level 1 recommendation in National Comprehensive Cancer Network (NCCN) guidelines, where it remains only a consideration in patients with ES-SCLC who have a response to systemic therapy (13). Indeed, great variability remains in the usage of thoracic RT in ES-SCLC (28).

Taken in meta-analysis, the 2 randomized studies examining thoracic RT suggest that thoracic RT is associated with improved overall survival (HR 08.1, 95\% CI: 0.69-0.96, $\mathrm{P}=0.014$ ) at the cost of a small increased risk of grade 3 esophageal toxicity (29). A secondary analysis of the CREST trial showed that the subgroup of patients with 2 or fewer metastases had significantly improved survival compared to patients with 3 or more sites of disease, in both the thoracic RT and the systemic therapy alone arms (30). Additionally, those patients with 2 or fewer sites of metastases had a significant benefit from thoracic RT, while thoracic RT for patients with 3 or more sites of metastasis did not impact survival. This indicates that volume of metastatic disease in ES-SCLC is an important prognostic factor, and may also predict which patients are most likely benefit from thoracic RT.

The RTOG 0937 trial hypothesized that treating patients with ES-SCLC with 4 or fewer sites of metastatic disease with consolidative RT to the thoracic disease and active sites of metastatic disease and PCI would delay progression and improve survival compared to a control arm which received PCI alone (31). In contrast to other studies which treated the thoracic disease with $30 \mathrm{~Gy}$ in 10 fractions, this trial recommended an aggressive consolidative RT dose of 45 Gy in 15 fractions to all active extracranial sites of disease. If normal tissue dose constraints were unable to be met with this dose, dose reduction to 30-40 Gy was allowed. This trial enrolled 97 patients between 2010 and 2015. The addition of consolidative RT delayed time to progression-at 3 months, $53.3 \%$ progressed in the PCI arm vs. $14.5 \%$ in the consolidative RT arm; however, at 12 months the rates of progression were $79.6 \%$ and $75 \%$ respectively. Unfortunately, 1-year OS showed no statistically significant difference at $60.1 \%$ for the PCI alone arm, and $50.8 \%$ for the PCI with consolidative RT arm (HR 1.44, $\mathrm{P}=0.21$ ). Toxicity was high with the addition of aggressive consolidative RT in this study, with rates of any grade 3 toxicity of $25 \%$ in the consolidative RT arm and $9.8 \%$ in the PCI alone arm. Additionally, the consolidative RT arm had 2 patients $(4.5 \%)$ with grade 4 toxicity and one patient $(2.3 \%)$ with grade 5 pneumonitis. Escalated thoracic doses contributing to cardiopulmonary toxicity may help to explain the lack of a survival benefit in the thoracic RT arm, negating any potential increase in tumor control. Furthermore, these data would suggest that radiation to extrathoracic metastases may not hold the same promise as it appears to in NSCLC $(32,33)$, and that thoracic radiation dose intensification beyond 30 Gy should be examined with caution in ES-SCLC.

\section{Immunotherapy and thoracic RT in ES-SCLC}

The use of immunotherapy may enhance efficacy of thoracic RT in ES-SCLC. Although the IMpower 133 trial did not allow thoracic RT, approximately $85 \%$ of patients had lung involvement, and $80 \%$ of patients had thoracic lymph node involvement. As only $2.5 \%$ of patients on the atezolizumab arm experienced a complete response, locoregional treatment could further improve outcomes in this population. While there are no randomized data evaluating the combination of immunotherapy and thoracic radiotherapy in ES-SCLC, the safety data and efficacy of the combination in the locally advanced non-small cell lung cancer (NSCLC) setting provides a foundation to build on. The PACIFIC trial evaluated patients with unresectable, 
locally advanced NSCLC who completed definitive concurrent chemoradiotherapy and were then randomized to the PD-L1 inhibitor durvalumab vs. placebo (34). The immunotherapy arm had significantly prolonged progression-free survival (PFS) (median 17.2 vs. 5.6 months) and improved 2-year OS (66.3\% vs. 55.6\%). Additionally, immunotherapy following definitive radiotherapy doses was well-tolerated, with only a modest increase in any grade pneumonitis (34\% for durvalumab vs. $25 \%$ for placebo), and a similar rate of grade $3-4$ pneumonitis $(3.4 \%$ vs. $2.6 \%$, respectively) (35). Other data indicate that the combination of thoracic radiotherapy and immunotherapy is safe and well-tolerated, in both patients with NSCLC (36-38) as well as SCLC (39). An analysis of 3 prospective studies combining immunotherapy and thoracic RT for patients with NSCLC or SCLC included 26 patients with ES-SCLC who received 45 Gy in 15 daily fractions to thoracic disease with concurrent chemotherapy and pembrolizumab, and 25 patients with limited stage SCLC (LS-SCLC) who received 45 Gy in 30 twice-daily fractions with concurrent chemotherapy and pembrolizumab (39). With an additional 27 patients with NSCLC treated with thoracic RT and concurrent pembrolizumab added to the analysis ( 78 total patients), there were seven total grade 4 events in four patients, only three of which were pulmonary-specific. The safety and efficacy of thoracic chemoradiation with immunotherapy will be formally tested in the upcoming NRG Oncology phase III study (LU005) of chemoradiation +/- atezolizumab in the LS-SCLC setting (NCT03811002) (40).

Additionally, the use of thoracic RT may enhance the effect of immunotherapy. Radiotherapy itself influences the immune system and its interactions with cancer cells and tumors, producing cytokines that recruit anti-tumor immune cells, increasing the exposure of tumor antigens, and improving cross-presentation of these antigens to the adaptive immune system (41-43). Preclinical data show evidence of a synergistic effect between radiotherapy and immunotherapy, leading to improved tumor control with a combination of RT and immunotherapy than with either therapy alone $(44,45)$. Additionally, cases of tumor regression outside of the radiation treatment field after radiotherapy is added to immunotherapy have been reported, termed the abscopal effect $(42,45)$. Although rare, the concurrent use of immunotherapy appears to improve the chances of an abscopal response (46).

This has led to enthusiastic investigation of the combination of radiotherapy and immunotherapy. One study of 39 patients with chemotherapy-refractory, metastatic NSCLC received palliative radiotherapy to one metastatic lesion (either $30 \mathrm{~Gy}$ in 5 fractions or 9 Gy in 3 fractions) with concurrent ipilimumab (41). There was an $18 \%$ overall radiographic response in the enrolled patients, with $31 \%$ of patients achieving disease control. Additionally, early immunologic changes following RT (increased interferon- $\beta$ and early changes in T-cells) were predictors of response, supporting the mechanistic role of radiotherapy in the improved immunotherapeutic response. Another study randomized 74 advanced NSCLC patients ( $>2$ nd line) to pembrolizumab \pm preceding SBRT to a single metastasis (3 fractions of 8 Gy). The PFS of the experimental arm was 6.4 vs. 1.8 months for the pembrolizumab monotherapy arm (HR 0.55, P=0.04) (47).

The available pre-clinical and clinical data suggest that a combination of immunotherapy with thoracic RT in the ES-SCLC population is likely to be safe, and could improve disease control, but these suggestions require further investigation. Many other prospective studies of the combination of immunotherapy and radiotherapy in SCLC are enrolling and under design.

\section{Prophylactic cranial RT in ES-SCLC}

Patients treated for SCLC have a high rate of developing brain metastases in the course of their disease, with approximately two-thirds of patients developing brain metastases by 2 years $(48,49)$. This led to the examination of PCI in patients with SCLC in effort to reduce the impact of brain metastases and improve survival. A meta-analysis of seven trials performed by Aupérin evaluated 987 patients with any stage of SCLC who had a complete response to chemotherapy reported that PCI significantly improved survival with an absolute survival benefit of $5.4 \%$ at 3 years (50). However, given the rarity of complete response in ES-SCLC to chemotherapy, the value of PCI in this setting remained unclear. Therefore the EORTC conducted a trial which randomized 286 patients with ES-SCLC who had any response to chemotherapy to PCI $v s$. observation (3). The PCI arm in this trial had a reduced incidence of symptomatic brain metastases by 1 year $[14.6 \%$ vs. 40.4\%; HR 0.27 (95\% CI: 0.16-0.44, $\mathrm{P}<0.001$ )] and improved 1-year OS [27.1\% vs. $13.3 \%$; HR 0.68 (95\% CI: $0.52-0.88), \mathrm{P}=0.003]$. Of note, routine brain MRI was not utilized for staging or follow-up in this trial, and cranial imaging was performed only if symptoms were suggestive of intracranial disease. As a result, there may have been patients with occult brain metastases at time of enrollment, 
which could have potentially skewed the results in favor of the PCI arm. This is an important limitation of this study. If mandatory screening MRI had been performed, the benefit of PCI may have been less than what was reported. If more frequent surveillance MRI of the brain had been performed, one could postulate that intracranial progression could have been detected earlier, potentially leading to higher rates (and efficacy) of salvage brain-directed RT.

Now in the era of routine MRI utilization, more recent data have called the efficacy of PCI in ES-SCLC into question. A multi-institutional Japanese study evaluated 224 patients with ES-SCLC who had any response to chemotherapy and a negative brain MRI prior to enrollment (51). These patients were randomized to PCI $v s$. routine MRI surveillance every 3 months for the first year, and 18 and 24 months after enrollment.

The study was terminated early due to futility on interim analysis. The PCI arm experienced a lower rate of brain metastases ( $40.1 \%$ at 18 months) compared to the surveillance arm (63.8\% at 18 months). Of the 77 patients in the surveillance arm who developed brain metastases, $83 \%$ received brain-directed salvage RT. In contrast to earlier studies, there was no significant difference in OS (median 11.6 months in the PCI arm vs. 13.7 months with observation; HR 1.27, 95\% CI: 0.96-1.68, P=0.094). Given these discordant data, additional data in a randomized trial to evaluate the value of PCI in SCLC (both ES-SCLC and LS-SCLC) vs. MRI surveillance is warranted.

To date, these data suggest that with routine MRI surveillance, patients with ES-SCLC may avoid PCI. This is an important consideration, as even the modest doses used with PCI (standardly 25 Gy in 10 fractions) still contribute to worsened neurocognitive outcomes and quality of life $(52,53)$. However, the impact of PCI on neurocognitive function may be ameliorated by use of memantine [N-methyl-D-aspartate (NMDA) receptor antagonist] in combination with hippocampal-avoiding whole brain RT (HA-WBRT) techniques. This was recently demonstrated in a large randomized trial (NRG Oncology CC001), showing an $11 \%$ absolute reduction in neurocognitive dysfunction via incorporation of HA-WBRT with memantine in patients with brain metastases (SCLC patients were excluded) (54). Furthermore, specific to PCI in SCLC, a small Spanish randomized phase III trial $(\mathrm{n}=60)$ showed marked reduction in the detriment to neurocognitive function as measured by delayed free recall and total free recall with incorporation of HA-WBRT techniques in PCI as compared to standard PCI (55).
NRG CC003 is currently enrolling, seeking to confirm these intriguing early results (in a randomized fashion) that suggest that HA-WBRT will reduce the neurocognitive and quality of life detriments associated with standard WBRT techniques in PCI (NCT02635009) (56).

\section{Immunotherapy and PCI}

Increased utilization of immunotherapy for ES-SCLC may further erode the potential use of PCI in this population. The available data from the IMpower-133 study did not include incidence of new brain metastasis, so it is unknown if atezolizumab impacted the rate of intracranial progression in this population. However, extrapolating the evidence from the PACIFIC trial in NSCLC, the addition of immunotherapy reduced the incidence of brain metastases in NSCLC (6.3\% vs. 11.8\%) (34). There is also evidence of CNS activity with immunotherapy in a study of ipilimumab/nivolumab in melanoma metastatic to the brain (57). If the inclusion of immunotherapy does reduce the incidence (or pace) of brain metastases presentation in ES-SCLC, then the rationale for upfront, routine PCI usage would be further decreased. While HA-WBRT reductions in neurotoxicity (if confirmed in PCI setting per NRG CC003) may favorably change the therapeutic ratio of PCI, the potential alteration in the natural history of ES-SCLC with immunotherapy, in combination with the availability for surveillance MRI, may result in decreasing enthusiasm for PCI in ES-SCLC. Whether radiographically apparent brain metastases (at diagnosis or during active MRI surveillance) can be appropriately treated with stereotactic radiosurgery also remains an open question [as under examination in the ongoing ENCEPHALON trial (NCT03297788)] (58).

\section{Future directions}

The incorporation of ICI into the upfront treatment of ES-SCLC presents opportunities to further leverage radiotherapy to improve outcomes for these patients. Unanswered questions include:

(I) Can ICI utilization render extrathoracic RT consolidation valuable [i.e., the previously negative RTOG 0937 (31) approach]?

(II) Could more routine utilization of consolidative chest RT further augment the systemic activity (and overall survival impact) of ICI?

(III) Does ICI further tip the scales away from routine 
PCI in ES-SCLC, towards a strategy of active MRI surveillance (59)?

(IV) For patients who develop brain metastases does ICI availability lend further rationale to the interest in stereotactic radiosurgery as opposed to standard WBRT (60)?

\section{Conclusions}

Immunotherapy has become a component of standard therapy in the front-line setting for ES-SCLC, and this will result in changing patterns of RT utilization. The addition of immunotherapy may increase enthusiasm for thoracic RT in patients who respond to systemic therapy. The combination of immunotherapy and thoracic radiotherapy is safe and well-tolerated to date in NSCLC, and may help to further consolidate locoregional disease as well as potentiate the immunotherapy effect at sites of extrathoracic metastases. Further studies are warranted to further investigate this strategy in ES-SCLC.

Additionally, immunotherapy may further decrease the use of PCI in ES-SCLC. Routine MRI surveillance has already supplanted the use of PCI for many practitioners, and the risk of neurocognitive toxicity may outweigh the benefit in this population despite the potential of hippocampal-avoiding WBRT. If immunotherapy further decreases the pace of brain metastases development in ES-SCLC, stereotactic radiosurgery may become a more readily-utilized alternative.

\section{Acknowledgements}

None.

\section{Footnote}

Conflicts of Interest: TJ Kruser has served on advisory board for AbbVie Inc., has consulted for Varian Medical Systems, and is on a speaker's bureau for AstraZeneca. TA Leal is a consultant/advisory board member for Roche-Genentech, Takeda, AstraZeneca, Novartis, AbbVie, Bristol-Myers Squibb, Bayer. EG Nesbit has no conflicts of interest to declare.

\section{References}

1. Horn L, Mansfield AS, Szczęsna A, et al. First-line atezolizumab plus chemotherapy in extensive-stage small- cell lung cancer. N Engl J Med 2018;379:2220-9.

2. Jeremic B, Shibamoto Y, Nikolic N, et al. Role of radiation therapy in the combined-modality treatment of patients with extensive disease small-cell lung cancer: a randomized study. J Clin Oncol 1999;17:2092-9.

3. Slotman B, Faivre-Finn C, Kramer G, et al. Prophylactic cranial irradiation in extensive small-cell lung cancer. $\mathrm{N}$ Engl J Med 2007;357:664-72.

4. Slotman BJ, van Tinteren H, Praag JO, et al. Use of thoracic radiotherapy for extensive stage small-cell lung cancer: a phase 3 randomised controlled trial. Lancet 2015;385:36-42.

5. D'amico D, Carbone D, Mitsudomi T, et al. High frequency of somatically acquired $\mathrm{p} 53$ mutations in small-cell lung cancer cell lines and tumors. Oncogene 1992;7:339-46.

6. Miller CW, Simon K, Aslo A, et al. p53 mutations in human lung tumors. Cancer Res 1992;52:1695-8.

7. George J, Lim JS, Jang SJ, et al. Comprehensive genomic profiles of small cell lung cancer. Nature 2015;524:47.

8. McGranahan N, Furness AJ, Rosenthal R, et al. Clonal neoantigens elicit $\mathrm{T}$ cell immunoreactivity and sensitivity to immune checkpoint blockade. Science 2016;351:1463-9.

9. Reck M, Bondarenko I, Luft A, et al. Ipilimumab in combination with paclitaxel and carboplatin as first-line therapy in extensive-disease-small-cell lung cancer: results from a randomized, double-blind, multicenter phase 2 trial. Ann Oncol 2013;24:75-83.

10. Riess JW, Lara PN Jr, Gandara DR. Theory meets practice for immune checkpoint blockade in small-cell lung cancer. J Clin Oncol 2016;34:3717.

11. Antonia SJ, López-Martin JA, Bendell J, et al. Nivolumab alone and nivolumab plus ipilimumab in recurrent small-cell lung cancer (CheckMate 032): a multicentre, open-label, phase 1/2 trial. Lancet Oncol 2016;17:883-95.

12. Hellmann MD, Ott PA, Zugazagoitia J, et al. Nivolumab (nivo) \pm ipilimumab (ipi) in advanced small-cell lung cancer (SCLC): First report of a randomized expansion cohort from CheckMate 032. American Society of Clinical Oncology, 2017.

13. NCCN Clinical Practice Guidelines in Oncology (NCCN Guidelines ${ }^{\circledR)}$ Small Cell Lung Cancer, Version 1.2019. Available online: Available online: https://www.nccn.org/ professionals/physician_gls/pdf/sclc.pdf

14. FDA grants nivolumab accelerated approval for third-line treatment of metastatic small cell lung cancer. Available 
online: https://www.fda.gov/Drugs/InformationOnDrugs/ ApprovedDrugs/ucm617370.htm

15. Chung HC, Lopez-Martin JA, Kao SCH, et al. Phase 2 study of pembrolizumab in advanced small-cell lung cancer (SCLC): KEYNOTE-158. J Clin Oncol 2018;36:8506.

16. Ott PA, Elez E, Hiret $\mathrm{S}$, et al. Pembrolizumab in patients with extensive-stage small-cell lung cancer: results from the phase Ib KEYNOTE-028 study. J Clin Oncol 2017;35:3823-9.

17. Reck M, Vicente D, Ciuleanu T, et al., editors. Efficacy and safety of nivolumab (nivo) monotherapy versus chemotherapy (chemo) in recurrent small cell lung cancer (SCLC): Results from CheckMate 331. ESMO ImmunoOncology Congress, 2018.

18. Pujol JL, Greillier L, Audigier-Valette C, et al. A Randomized Non-Comparative Phase 2 Study of AntiProgrammed Cell Death-Ligand 1 Atezolizumab or Chemotherapy as Second-Line Therapy in Patients with Small Cell Lung Cancer: Results from the IFCT-1603 Trial. J Thorac Oncol 2019;14:903-13.

19. Passiglia F, Novello S. Immunotherapy in first line for extensive-stage small-cell lung cancer: another piece is going to fill the puzzle? Ann Transl Med 2018;6:S120.

20. Bristol-Myers Squibb Announces CheckMate -451 Study Did Not Meet Primary Endpoint of Overall Survival with Opdivo Plus Yervoy Vs. Placebo as A Maintenance Therapy in Patients with Extensive-Stage Small Cell Lung Cancer After Completion of First-Line. Available online: https://news.bms.com/press-release/corporatefinancialnews/bristol-myers-squibb-announces-checkmate-451study-did-not-mee

21. Califano R, Każarnowicz A, Karaseva N, et al. 490 IMpower133: Patient-reported outcomes (PROs) in a ph1/3 study of first-line (1L) atezolizumab (atezo)+ carboplatin+ etoposide (CP/ET) in extensive-stage SCLC (ES-SCLC). Ann Oncol 2018;29:mdy486.

22. FDA approves atezolizumab for extensive-stage small cell lung cancer. Available online: https:/www.fda.gov/Drugs/ InformationOnDrugs/ApprovedDrugs/ucm633814.htm

23. Mansfield AS, Liu SV, Szczęsna A, et al., editors. IMpower133: Primary efficacy and safety + CNSrelated adverse events in a $\mathrm{Ph} 1 / 3$ study of first-line (1L) atezolizumab (atezo) + carboplatin + etoposide in extensive-stage SCLC (ES-SCLC). American Association for Cancer Research, 2019.

24. Früh M, De Ruysscher D, Popat S, et al. Small-cell lung cancer (SCLC): ESMO Clinical Practice Guidelines for diagnosis, treatment and follow-up. Ann Oncol 2013;24:vi99-105.

25. Giuliani ME, Atallah S, Sun A, et al. Clinical outcomes of extensive stage small cell lung carcinoma patients treated with consolidative thoracic radiotherapy. Clin Lung Cancer 2011;12:375-9.

26. Zhu H, Zhou Z, Wang Y, et al. Thoracic radiation therapy improves the overall survival of patients with extensivestage small cell lung cancer with distant metastasis. Cancer 2011;117:5423-31.

27. Yee D, Butts C, Reiman A, et al. Clinical trial of postchemotherapy consolidation thoracic radiotherapy for extensive-stage small cell lung cancer. Radiother Oncol 2012;102:234-8.

28. Ciammella P, Timon G, Bruni A, et al. Radiation therapy in small cell lung cancer: a national Italian survey. Radiol Med 2018;123:554-60.

29. Palma DA, Warner A, Louie AV, et al. Thoracic Radiotherapy for Extensive Stage Small-Cell Lung Cancer: A Meta-Analysis. Clin Lung Cancer 2016;17:239-44.

30. Slotman BJ, Faivre-Finn C, van Tinteren H, et al. Which patients with ES-SCLC are most likely to benefit from more aggressive radiotherapy: A secondary analysis of the Phase III CREST trial. Lung Cancer 2017;108:150-3.

31. Gore EM, Hu C, Sun AY, et al. Randomized phase II study comparing prophylactic cranial irradiation alone to prophylactic cranial irradiation and consolidative extracranial irradiation for extensive-disease small cell lung cancer (ED SCLC): NRG Oncology RTOG 0937. J Thorac Oncol 2017;12:1561-70.

32. Iyengar P, Wardak Z, Gerber DE, et al. Consolidative radiotherapy for limited metastatic non-small-cell lung cancer: A phase 2 randomized clinical trial. JAMA Oncol 2018;4:e173501.

33. Gomez DR, Blumenschein GR Jr, Lee JJ, et al. Local consolidative therapy versus maintenance therapy or observation for patients with oligometastatic non-smallcell lung cancer without progression after first-line systemic therapy: a multicentre, randomised, controlled, phase 2 study. Lancet Oncol 2016;17:1672-82.

34. Antonia SJ, Villegas A, Daniel D, et al. Overall survival with durvalumab after chemoradiotherapy in stage III NSCLC. N Engl J Med 2018;379:2342-50.

35. Antonia SJ, Villegas A, Daniel D, et al. Durvalumab after chemoradiotherapy in stage III non-small-cell lung cancer. N Engl J Med 2017;377:1919-29.

36. Shaverdian N, Lisberg AE, Bornazyan K, et al. Previous radiotherapy and the clinical activity and toxicity of pembrolizumab in the treatment of non-small-cell lung 
cancer: a secondary analysis of the KEYNOTE-001 phase 1 trial. Lancet Oncol 2017;18:895-903.

37. Bang A, Wilhite TJ, Pike LR, et al. Multicenter evaluation of the tolerability of combined treatment with PD-1 and CTLA-4 immune checkpoint inhibitors and palliative radiation therapy. Int J Radiat Oncol Biol Phys 2017;98:344-51.

38. Ahmed K, Grass G, Creelan B, et al. Tolerability and Safety of Thoracic Radiation and Immune Checkpoint Inhibitors Among Patients with Lung Cancer. Int J Radiat Oncol Biol Phys 2017;98:224.

39. Verma V, Cushman TR, Selek U, et al. Safety of Combined Immunotherapy and Thoracic Radiation Therapy: Analysis of 3 Single-Institutional Phase I/II Trials. Int J Radiat Oncol Biol Phys 2018;101:1141-8.

40. Chemoradiation With or Without Atezolizumab in Treating Patients With Limited Stage Small Cell Lung Cancer. 2019. Available online: https://clinicaltrials.gov/ ct2/show/NCT03811002

41. Formenti SC, Rudqvist NP, Golden E, et al. Radiotherapy induces responses of lung cancer to CTLA-4 blockade. Nat Med 2018;24:1845.

42. Demaria $\mathrm{S}, \mathrm{Ng} \mathrm{B}$, Devitt $\mathrm{ML}$, et al. Ionizing radiation inhibition of distant untreated tumors (abscopal effect) is immune mediated. Int J Radiat Oncol Biol Phys 2004;58:862-70.

43. Golden EB, Chhabra A, Chachoua A, et al. Local radiotherapy and granulocyte-macrophage colonystimulating factor to generate abscopal responses in patients with metastatic solid tumours: a proof-of-principle trial. Lancet Oncol 2015;16:795-803.

44. Deng L, Liang H, Burnette B, et al. Irradiation and antiPD-L1 treatment synergistically promote antitumor immunity in mice. J Clin Invest 2014;124:687-95.

45. Dovedi SJ, Cheadle EJ, Popple AL, et al. Fractionated Radiation Therapy Stimulates Antitumor Immunity Mediated by Both Resident and Infiltrating Polyclonal T-cell Populations when Combined with PD-1 Blockade. Clin Cancer Res 2017;23:5514-26.

46. Postow MA, Callahan MK, Barker CA, et al. Immunologic correlates of the abscopal effect in a patient with melanoma. N Engl J Med 2012;366:925-31.

47. Theelen W, Peulen H, Lalezari F, et al. Randomized phase II study of pembrolizumab after stereotactic body radiotherapy (SBRT) versus pembrolizumab alone in patients with advanced non-small cell lung cancer: The PEMBRO-RT study. American Society of Clinical Oncology, 2018.
48. Arriagada R, Le Chevalier T, Borie F, et al. Prophylactic cranial irradiation for patients with small-cell lung cancer in complete remission. J Natl Cancer Inst 1995;87:183-90.

49. Nugent JL, Bunn PA Jr, Matthews MJ, et al. CNS metastases in small cell bronchogenic carcinoma. Increasing frequency and changing pattern with lengthening survival. Cancer 1979;44:1885-93.

50. Aupérin A, Arriagada R, Pignon JP, et al. Prophylactic cranial irradiation for patients with small-cell lung cancer in complete remission. N Engl J Med 1999;341:476-84.

51. Takahashi T, Yamanaka T, Seto T, et al. Prophylactic cranial irradiation versus observation in patients with extensive-disease small-cell lung cancer: a multicentre, randomised, open-label, phase 3 trial. Lancet Oncol 2017;18:663-71.

52. Le Péchoux C, Laplanche A, Faivre-Finn C, et al. Clinical neurological outcome and quality of life among patients with limited small-cell cancer treated with two different doses of prophylactic cranial irradiation in the intergroup phase III trial (PCI99-01, EORTC 2200308004, RTOG 0212 and IFCT 99-01). Ann Oncol 2011;22:1154-63.

53. Slotman BJ, Mauer ME, Bottomley A, et al. Prophylactic cranial irradiation in extensive disease small-cell lung cancer: short-term health-related quality of life and patient reported symptoms-results of an international phase III randomized controlled trial by the EORTC radiation oncology and lung cancer groups. J Clin Oncol 2009;27:78.

54. Gondi V, Pugh S, Laack N, et al. Preservation of Neurocognitive Function (NCF) with Hippocampal Avoidance During Whole-Brain Radiotherapy (WBRT) for Brain Metastases: Preliminary Results of Phase III Trial NRG Oncology CC001. Neuro Oncol 2018;20:vi172.

55. Couñago F, López J, Calvo P, et al. OC-0498: Randomized phase III trial of PCI with or without hippocampal avoidance for SCLC. Radiat Oncol 2018;127:S256-7.

56. Whole-Brain Radiation Therapy With or Without Hippocampal Avoidance in Treating Patients With Limited Stage or Extensive Stage Small Cell Lung Cancer. Available online: https://clinicaltrials.gov/ct2/show/ NCT02635009

57. Tawbi HA, Forsyth PA, Algazi A, et al. Combined Nivolumab and Ipilimumab in Melanoma Metastatic to the Brain. N Engl J Med 2018;379:722-30.

58. Bernhardt D, Hommertgen A, Schmitt D, et al. Whole brain radiation therapy alone versus radiosurgery for 
patients with 1-10 brain metastases from small cell lung cancer (ENCEPHALON Trial): study protocol for a randomized controlled trial. Trials 2018;19:388.

59. Rusthoven CG, Kavanagh BD. Prophylactic cranial irradiation (PCI) versus active MRI surveillance for small cell lung cancer: the case for equipoise. J Thorac Oncol 2017;12:1746-54.

60. Robin TP, Jones BL, Amini A, et al. Radiosurgery alone is associated with favorable outcomes for brain metastases from small-cell lung cancer. Lung Cancer 2018;120:88-90.

Cite this article as: Nesbit EG, Leal TA, Kruser TJ. What is the role of radiotherapy for extensive-stage small cell lung cancer in the immunotherapy era? Transl Lung Cancer Res 2019;8(Suppl 2):S153-S162. doi: 10.21037/tlcr.2019.05.01 